
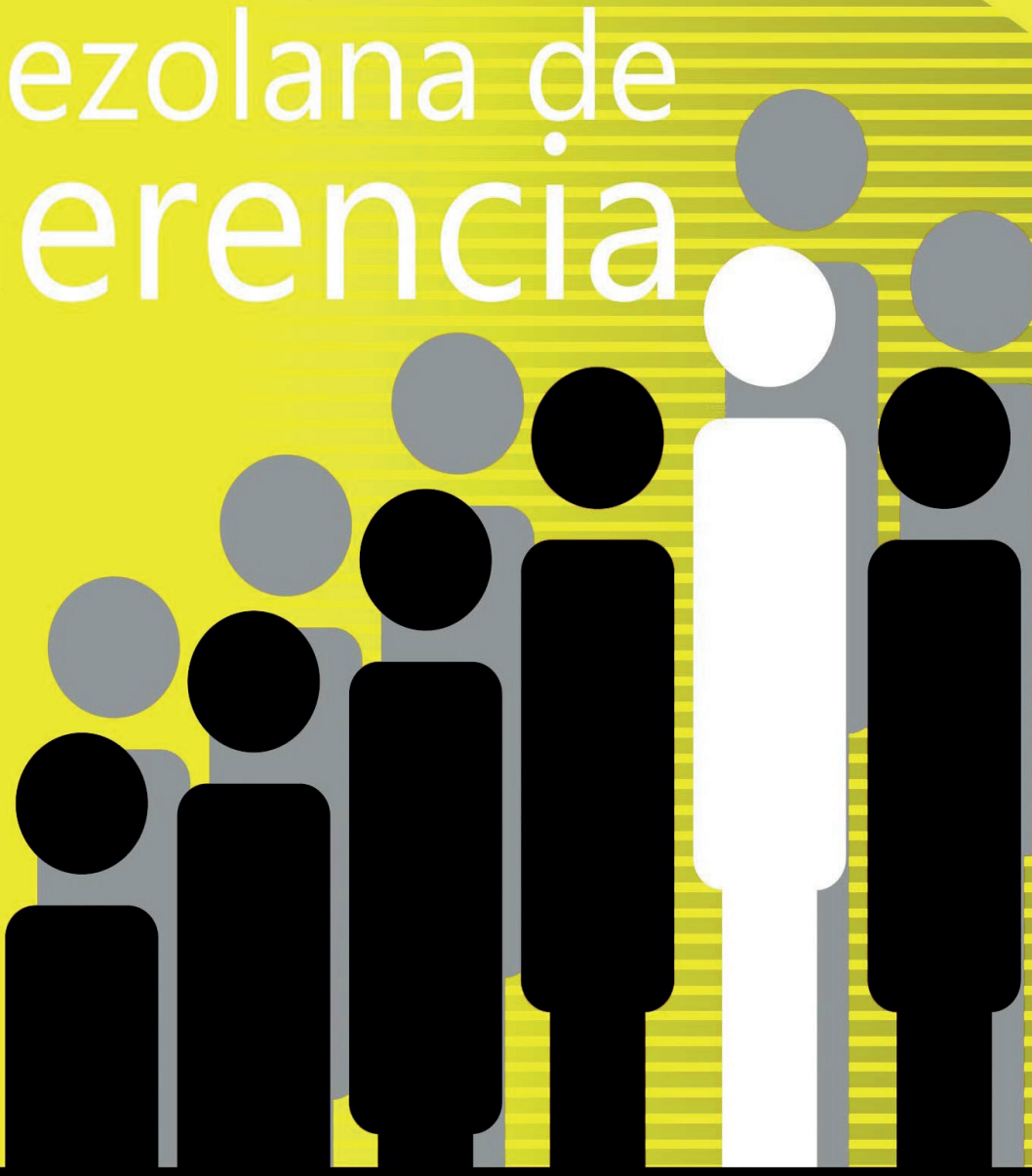


\title{
Capacidad de Absorción e Innovación en empresas industriales manufactureras en Uruguay
}

\author{
Silveira, Luis* \\ Horta, Roberto* \\ Francia, Heber ${ }^{* * *}$
}

\section{Resumen}

Las innovaciones permiten a las empresas responder a los desafíos del mercado, obtener ventajas competitivas sostenibles y promover el crecimiento económico. El objetivo de esta investigación es determinar si la capacidad de absorción aumenta la probabilidad de que una empresa sea innovadora. Para ello se aplicaron diferentes modelos logit a los micro-datos de la Encuesta de Actividades de Innovación de las empresas industriales manufactureras uruguayas. Los resultados permiten concluir que la capacidad de absorción es un elemento importante para explicar la probabilidad de que una firma sea innovadora. Inciden en este sentido la existencia de unidades formales de I+D en la firma y la magnitud del cociente entre los empleados ocupados en tareas de I+D sobre el total de empleados de la firma.

Palabras clave: Capacidad de absorción; Innovación; Industria manufacturera uruguaya.

Recibido: 13.10 .20 aceptado: 15.02 .21

* Doctor en Competitividad Empresarial y Desarrollo Económico, Universidad de Deusto (España). Magíster en Economía y Regulación de los Servicios Públicos, Universitat de Barcelona (España). Magíster en Gerencia de Empresas de Telecomunicaciones, Universidad ORT (Uruguay). Economista, Universidad de la República (Uruguay). Investiga en temas de competitividad, innovación y clusters. Es investigador en el Instituto de Competitividad de la Universidad Católica del Uruguay y Profesor Asociado en la misma universidad. ORCID: https://orcid.org/0000-0001-9506-256X.

** Doctor en Economía y Dirección de Empresas por la Universidad de Deusto (San Sebastián, España) y Economista - Licenciado en Economía por la Universidad de la República (Montevideo, Uruguay). Investiga en temas de competitividad, innovación e internacionalización de empresas. Es investigador en el Instituto de Competitividad de la Universidad Católica del Uruguay. Integra el Sistema Nacional de Investigadores en Uruguay. ORCID: https://orcid.org/0000-0001-5138-2377.

*** Doctor en Ciencias Económicas y Empresariales con especialidad en Economía Aplicada: Universidad Nacional de Educación a Distancia (UNED) - Madrid - España, Máster en Estadística Matemática: Centro Interamericano de Enseñanza de Estadística (CIENES). Santiago - Chile y Economista - Licenciado en Economía por la Universidad de la República (Montevideo, Uruguay). Profesor de economía de la Universidad de Montevideo. ORCID: http://orcid.org/0000-0001-5507-0215 


\title{
Absorption Capacity and Innovation in Industrial Manufacturing Companies in Uruguay
}

\begin{abstract}
The innovation that companies make allow them to respond to market challenges, obtain sustainable competitive advantages over time and promote economic growth. The objective of this research is to determine if absorption capacity increases the probability that a company will be innovative. For this, different logit models were applied to the micro-data of the Survey of Innovation Activities of Uruguayan industrial manufacturing companies. The results allow us to conclude that absorption capacity is an important element to explain the probability that a firm is innovative. In this sense, the existence of formal R\&D units in the firm and the magnitude of the ratio between the employees engaged in R\&D tasks over the total number of firm employees have an impact on this.
\end{abstract}

Keywords: Absorption capacity; Innovation; Uruguayan manufacturing industry.

\section{Introducción}

Los procesos de innovación que realizan las empresas les permiten responder a los desafíos del mercado, obtener ventajas competitivas sostenibles en el tiempo (Vermeulen, 2004) y promover el crecimiento económico (Cheng y Tao, 1999; González-Campo y Hurtado Ayala, 2014). Por ello, la innovación es un tema que tiene mucho interés, tanto para los académicos como para los hacedores de políticas públicas. Esto se debe a que la innovación es un proceso complejo a través del cual las firmas transforman conocimientos en valor agregado. Una de las definiciones de innovación más reconocidas es la desarrollada por la Organización para la Cooperación y el Desarrollo Económicos (OCDE) en el "Manual de Oslo" que expresa que: "una innovación es la introducción de un nuevo, o significativamente mejorado, producto (bien o servicio), de un proceso, de un nuevo método de comercialización o de un nuevo método organizativo, en las practicas internas de la empresa, la organización del lugar de trabajo o las relaciones exteriores" (OCDE, 2005: 56).

Para las economías en desarrollo, la innovación puede contribuir a que la industria manufacturera incorpore cada vez más valor a las materias primas y de esa manera logre superar la competencia en precios y la dependencia del comercio exterior basada en los commodities. A su vez, una mayor innovación a nivel de las firmas, en dicho sector industrial, puede ayudar a sostener un incremento sistemático de salarios sin afectar los niveles de competitividad. 
Finalmente, procesos crecientes de innovación pueden evitar el deterioro de los términos de intercambio y los desequilibrios externos, permitiendo un mejor aprovechamiento de los recursos naturales (Lugones, Suarez y Gregorini (2007).

Esa situación no es diferente en el caso de Uruguay (Camacho, et al, 2010: 97), por lo que comprender, cómo se verifican los procesos de innovación en las empresas uruguayas, se vuelve crítico para la correcta planificación y aplicación de políticas de incentivos.

Una de las razones por las cuales las empresas tienen dificultades para innovar puede tener que ver con la poca capacidad de absorción que presentan. En ese sentido, la relación entre capacidad de absorción e innovación ha venido siendo investigada desde hace relativamente poco tiempo (Rodríguez, et al, 2017). Por ejemplo, de acuerdo con Kostopoulos et al, (2011), la capacidad de absorción se puede considerar un medio para realizar una mayor actividad innovadora y para lograr la trasformación del conocimiento externo en resultados, situación que tiende a reflejarse en un mayor nivel de innovación que presentan las empresas.

Al ser Latinoamérica la región con la menor cantidad de estudios de investigación sobre la capacidad de innovación de las firmas (Aguilera, et al, 2017), son necesarias investigaciones adicionales en la materia, que ayuden a las empresas a afrontar los retos actuales de la economía global (Del Carpio y Miralles, 2018). Ello debería ayudar también en el proceso de toma de decisiones de inversión en innovación, en el desarrollo económico y en la creación de nuevos puestos de trabajo, en el crecimiento de las exportaciones de bienes y servicios con mayor valor agregado $y$, en consecuencia, en mejores productos y servicios para los consumidores (Brenes et al, 2016).

A partir de los comentarios anteriores, el objetivo central del estudio consiste en determinar, para el caso de las empresas del sector de la industria manufacturera en Uruguay, si la capacidad de absorción aumenta la probabilidad de que la empresa sea innovadora, definiéndose ésta como aquella empresa que realizó al menos un tipo de innovación en el periodo de estudio. Adicionalmente, la investigación busca establecer cuáles son las características de la capacidad de absorción que realmente tienen efecto en las innovaciones realizadas.

Se realizó una revisión sistemática de bibliografía que estudia la misma problemática que se postula en el objetivo de la presente investigación. Para el análisis empírico se utilizaron los micro-datos de la última Encuesta de Actividades de Innovación (EAI) de la Agencia Nacional de Investigación e Innovación (ANII) de Uruguay ${ }^{1}$ y se aplicaron a dichos micro-datos diferentes modelos de regresión de respuesta dicotómica logit.

1 La última EAI publicada por la ANII corresponde al período 2013-2015. Se prevé que los resultados de la encuesta para el periodo 2016-2019 recién se publique y esté disponible a finales de 2021. 

Uruguay

Para la investigación se seleccionaron empresas de la encuesta que integran el sector de la industria manufacturera y que se desempeñan en los rubros de Alimentos, bebidas y tabaco, Textiles, prendas de vestir y cuero, Madera, papel y derivados, Refinación de petróleo, Químicos, caucho y plástico, Metálicos básicos, Maquinaria y equipo y Material de transporte. Elegir las firmas de la industria manufacturera como objeto de estudio de esta investigación tiene dos razones principales. La primera deriva del hecho de que la industria manufacturera en Uruguay significa un porcentaje muy importante del Producto Interno Bruto (PIB) del país, 19\% para el año 2019 según datos del Banco Central del Uruguay (BCU) (BCU, 2019) y de que la agroindustria es clave para incorporar valor a los productos tradicionales que produce el país (carne, cereales, celulosa). La segunda es que los autores han estado trabajando y se han especializado en este sector de la economía en diversas investigaciones a través de los años (Horta, Silveira y Camacho, 2015; Horta et al, 2020).

Por último, respecto a los resultados, se debe resaltar que todos los modelos logit utilizados, demostraron tener una muy buena bondad de ajuste ya que estimaron bien, como mínimo el $75 \%$ de las observaciones. Dichos resultados permiten concluir que la capacidad de absorción es un elemento importante para explicar la probabilidad de que una firma del sector a estudio sea innovadora. En este sentido, inciden de forma favorable que la firma cuente con unidades formales dedicadas a la investigación y desarrollo (I+D) en su estructura organizativa y la cantidad relativa de empleados ocupados en tareas de I+D con la que cuenten.

\section{Capacidad de Absorción e Innovación}

El estudio de las capacidades dinámicas permite comprender cómo las empresas administran sus recursos $y$ competencias para ajustarlo a las nuevas condiciones del entorno en el cual se desempeñan. Éstas son definidas como la combinación de recursos difíciles de imitar que permiten reconfigurar los recursos ante la dinámica del mercado generando ventajas competitivas de manera sostenible. Entre esos recursos que deben administrar las firmas, destacan la capacidad de aprender y de innovar para poder competir exitosamente en el mercado (Carpio y Afcha, 2020). Sánchez y Acosta (2020) agregan la capacidad de resiliencia empresarial como variable mediadora entre el aprendizaje tecnológico y la competitividad.

Los factores que determinan que una firma pueda innovar son diversos y suelen variar con el tiempo, pero dado que el proceso de innovación puede considerarse como un continuo proceso de aprendizaje donde las empresas no solo aprenden de su propia experiencia, sino que también adquieren información y conocimiento de diversas fuentes externas, para poder llevar adelante exitosamente dicho proceso es clave acceder adecuadamente a esa información (González-Campo y Hurtado Ayala, 2014).

Un aspecto muy importante, que condiciona muchas veces la posibilidad de acceder a información clave para poder innovar, es que las empresas cuenten con capacidad de absorción del conocimiento. Esto se debe a que muchas veces, a pesar de que el conocimiento externo a la firma es de dominio público, no es fácil de asimilar y 
por lo tanto no es igualmente absorbido y explotado por las firmas (Fabrizio, 2009; Kostopoulos et al, 2011; Ali, Seny y Sarstedt, 2016; De Macedo et al, 2016; Solís, Zerón y Sánchez, 2019; Yaseen, 2020).

La capacidad de absorción fue definida originalmente por Cohen $y$ Levinthal (1990) como la capacidad de una empresa de reconocer el valor de nueva información externa, asimilarla y aplicarla con fines comerciales. Además, estos autores afirman que la habilidad de explotar el conocimiento externo a la empresa es un componente crítico de la capacidad de innovación Existen otras conceptualizaciones tempranas del concepto. Por ejemplo. Mowery y Oxley (1995) consideran que la capacidad de absorción es un conjunto de habilidades necesarias para gestionar el componente tácito del conocimiento que se desea transferir con el fin de mejorar la adquisición de conocimiento externo. Lane y Lubatkin (1998) conceptualizan la capacidad de absorción de una organización de forma relativa, es decir, como la habilidad que tiene una empresa para asimilar y aplicar el conocimiento proveniente de otra empresa. Dyer y Singh (1998) definen la capacidad de absorción como un proceso repetitivo de intercambio que origina rentas relacionales 0 beneficios producidos por los procesos de interacción entre diferentes socios y miembros de una organización (González-Campo y Hurtado Ayala, 2014).

En el año 2002, Zahra y George presentaron una reconceptualización del concepto introducido por Cohen y Levinthal en 1990. Dichos autores definen la capacidad de absorción como "un set o conjunto de rutinas y procesos organizacionales, a través de los cuales las firmas adquieren, asimilan, transforman y explotan conocimientos para crear una organización con capacidad dinámica" (Zahra y George, 2002: 186). Posteriores revisiones del concepto fueron realizadas por Jansen, Van den Bosch y Volberda (2005), Lane, Koka y Patthak (2006) y Volverda, Foss y Lyles (2010). Por ejemplo, Lane et al, (2006) definen la capacidad de absorción como una consecuencia de la innovación anterior y de las capacidades que tiene la empresa para resolver problemas.

De acuerdo con Rodríguez, et al, (2017), la capacidad de absorción para Zahra y George (2002) está constituida por dos subconjuntos de procesos, aparentemente aislados, pero que se les considera complementarios por tener funciones independientes dentro de la organización. Dichos subconjuntos de procesos son: la capacidad de absorción potencial y la capacidad de absorción realizada. El primero incluye los procesos (o etapas) de adquisición y asimilación del nuevo conocimiento externo. El segundo incluye la transformación y explotación de dicho conocimiento. Según el artículo de Leal-Rodríguez et al, (2014), esta clasificación por etapas ha sido muy utilizada para captar este fenómeno.

La etapa de adquisición consiste en la capacidad de una empresa para identificar y adquirir el conocimiento externo que es crítico para sus operaciones. Esta etapa tiene tres atributos que pueden influir en la capacidad de absorción: intensidad, velocidad y dirección. La intensidad y la velocidad de los esfuerzos de una empresa para identificar y reunir los conocimientos necesarios pueden determinar la calidad de la capacidad de adquisición de una firma. La dirección de la acumulación de conocimiento también puede influir en los caminos 
que sigue la empresa en la obtención de conocimiento externo (Zahra y George, 2002: 189). Por otra parte, la asimilación se refiere a las rutinas y procesos de la firma que le permiten analizar, procesar, interpretar y comprender la información externa obtenida (Zahra y George, 2002: 189). Por último, la transformación y explotación, es la capacidad de una empresa para desarrollar y perfeccionar las rutinas y procesos que facilitan la combinación de los conocimientos existentes, con los nuevos recién adquiridos y así poder asimilarlos correctamente (Zahra y George, 2002: 190).

La capacidad de absorción y la innovación son conceptos que se relacionan debido a que, como ya se expresó, la posibilidad de innovar de una firma puede estar determinada por la adecuada adquisición de conocimientos, en general complejos. Para Carter (1989) (citado en Giuliani, 2005), la capacidad de absorción y la capacidad de innovar están directamente relacionadas ya que las firmas tienen más incentivo a requerir ayuda tecnológica cuando perciben que son capaces de decodificar y aplicar el conocimiento recibido. Expósito-Langa, Molina-Morales y Capo-Vicedo (2011) expresan que, a mayor capacidad de absorción de la firma, mayor es la posibilidad de asimilar y explotar el conocimiento adquirido.

En lo que refiere a la relación entre la capacidad de absorción y el tipo de innovación que ésta puede afectar, Schmidt y Rammer (2006) encontraron que las empresas que tenían mayor capacidad de absorción tienen más posibilidades de realizar los cuatro tipos de innovaciones (producto, procesos, organización o comercialización). Rangus y Slavec (2017) analizando una muestra de 421 empresas industriales y de servicios, encontraron que el nivel de capacidad de absorción tiene una influencia directa en las innovaciones en producto y en procesos.

Ali y Park (2017) analizado una muestra de 347 compañías industriales coreanas que presentaban niveles elevados de capacidad de absorción encontraron que eran empresas con niveles altos de innovaciones en producto y en proceso. Calero-Medina y Noyons (2008) encontraron que la relación entre capacidad de absorción y las innovaciones en organización es significativa. Además, Chen y Chang (2012) encontraron que, a mayor nivel de capacidad de absorción en la empresa, mayor es el aumento del grado de innovación en organización. En cuanto a cuál es la etapa más importante en la capacidad de absorción y su relación con la innovación, Expósito-Langa et al, (2011) concluyen que de las cuatro etapas de la capacidad de absorción desarrolladas por Zahra y George (2002), la más significativa es la etapa de adquisición.

La capacidad de absorción también está ligada al sistema de aprendizaje colectivo y conocimiento inherente a las firmas que pertenezcan a una red. O sea, a la posibilidad de que los flujos de conocimiento que interconectan a las empresas en red sean aprovechados de forma adecuada, los cuales pueden ser fundamentales a la hora de llevar adelante actividades de innovación (Heijs, 2010). Estudios empíricos demuestran que firmas con una amplia capacidad de absorción se benefician más de la presencia de flujos externos de conocimientos que aquellas que no la tienen (Escribano, Fosfuri y Tribó, 2009). Eso es así, ya sea porque la empresa con mayor capacidad de absorción puede identificar más y mejores conocimientos, 
o porque los puede explotar de una manera más eficiente.

Al ser la capacidad de absorción del conocimiento un elemento clave en el aprendizaje colectivo y por lo tanto en el proceso de innovación, se han propuesto diversas formas para tratar de incrementarla y mejorarla. Entre ellas, se destacan la inversión interna en recursos como ser las inversiones en $I+D$, las inversiones en capacitación de los empleados, así como en soluciones que tienen que ver con la promoción de vínculos al exterior de la firma y la formación de redes de intercambio de conocimientos (Giuliani, 2005; Morrison, 2008; Visser y Atzema, 2008; Lazaric, Longhi y Thomas, 2008; Jong y Freel, 2010).

En resumen, dado que la posibilidad de acceder e internalizar información es fundamental para que las empresas puedan innovar y así poder competir exitosamente en el mercado, contar con capacidad de absorción del conocimiento parece ser un factor clave para lograrlo. En este sentido, investigar la relación entre capacidad de absorción e innovación es importante, no solo para comprender mejor el fenómeno, sino también para poder ayudar a diseñar mejores políticas públicas que la incentiven.

\section{Consideraciones metodológicas}

El estudio empírico de esta investigación, donde se define la capacidad de absorción como un constructo multidimensional, es similar al desarrollado por diversas investigaciones (Vega-Jurado, et al, 2005; Cepeda-Carrión, Cegarra-Navarro y Jiménez-Jiménez, 2012; MaynezGuaderrama, Cavazos-Arroyo y Nuño- de la Parra, 2012; Ramadhan, Hussain, y al-Hajji ,2013; González-Campo y Hurtado Ayala, 2014),

Para realizar la investigación se utilizó la Encuesta de Actividades de Innovación (EAI) a las empresas industriales manufactureras en Uruguay para el período 2013-2015. En concreto, se utilizaron los micro-datos de 931 empresas industriales, de las cuales 721 eran pymes. El análisis de los microdatos se realizó aplicando un estudio de estadística descriptiva y un modelo econométrico de regresión logística (logit). Esto se debe a que todas las regresiones planteadas tienen como variable dependiente variables que son discretas (dicotómicas). El modelo utiliza variables que captan la innovación (como variable dependiente) y como variables independientes, aspectos que refieren a la capacidad de absorción y a algunas características de las firmas estudiadas (como variables de control). Las variables seleccionadas se detallan en el siguiente apartado.

Las variables que recogen las innovaciones realizadas por las empresas y que se seleccionaron para el modelo fueron:

- "Innovación tecnológica en

producto: es la introducción al mercado de un producto (bien o servicio) tecnológicamente nuevo (cuyas características tecnológicas 0 usos previstos difieran significativamente de los productos anteriores de la empresa) o significativamente mejorados (previamente existente pero cuyo desempeño ha sido perfeccionado o mejorado en gran medida).

- Innovación tecnológica en procesos: es la adopción de métodos de producción nuevos o significativamente mejorados. 


\begin{abstract}
Pueden permitir producir o entregar productos (bienes 0 servicios) tecnológicamente nuevos o mejorados, que no pueden producirse o entregarse utilizando métodos de producción convencionales, o bien aumentar significativamente la eficiencia de producción o entrega de productos existentes.
\end{abstract}

- Innovación en técnicas de organización: es la introducción de cambios en las formas de organización y gestión del establecimiento o local, cambios en la organización y administración del proceso productivo, incorporación de estructuras organizativas modificadas significativamente e implementación de orientaciones estratégicas nuevas o sustancialmente modificadas.

- Innovación en técnicas de comercialización: es la introducción de métodos para la comercialización de productos (bienes o servicios) nuevos, de nuevos métodos de entrega de productos existentes o de cambios de empaque o embalaje" (ANII, 2015: 9-10).

Para identificar la capacidad de absorción de las firmas se seleccionaron las variables teniendo en cuenta su disponibilidad en la encuesta y que además reflejaran de la mejor forma la teoría desarrollada por Zahra y George (2002):2
- Para la etapa de adquisición se seleccionaron las siguientes dos variables: la inversión en actividades de I+D (interna y externa) respecto a las ventas totales de la firma y la participación en redes. Por ejemplo, Cohen y Levinthal (1990); Stock, Greis y Fischer (2001); Tsai (2001); Oltra y Flor (2003); Belderbos, et al, (2004); Zahra y Hayton (2008) proponen la intensidad de I+D (gasto en $\mathrm{I}+\mathrm{D}$ dividido por ventas anuales).

- Respecto a la etapa de asimilación se seleccionaron dos variables: total de ocupados en actividades de I+D respecto al total de empleados de la firma y la existencia de unidades formales de $I+D$ en la firma. En este sentido, por ejemplo, se puede señalar que Luo (1997) utiliza en su investigación el primer tipo de variable, concretamente la cantidad del personal técnico y profesional dividido por el total del número de empleados de la organización.

- Para la etapa de transformación se seleccionó una variable: gastos de formación de personal para innovar respecto a las ventas anuales de la firma. La elección se basa en Petroni y Panciroli (2002), autores que proponen el esfuerzo de formación de personal (gastos de formación de personal sobre las ventas anuales) para esta fase.

La información anterior se resume en la siguiente tabla 1:

Al carecer de datos sobre patentes, ya que la encuesta de la ANII no incluye preguntas que traten de recabar información el respecto, no se toma en cuenta la etapa de explotación en la presente investigación. 
pp. $725-744$

Revista Venezolana de Gerencia, Año 26 No. 94, 2021

\section{Tabla 1 \\ Constructo Capacidad de Absorción (CAPABS)}

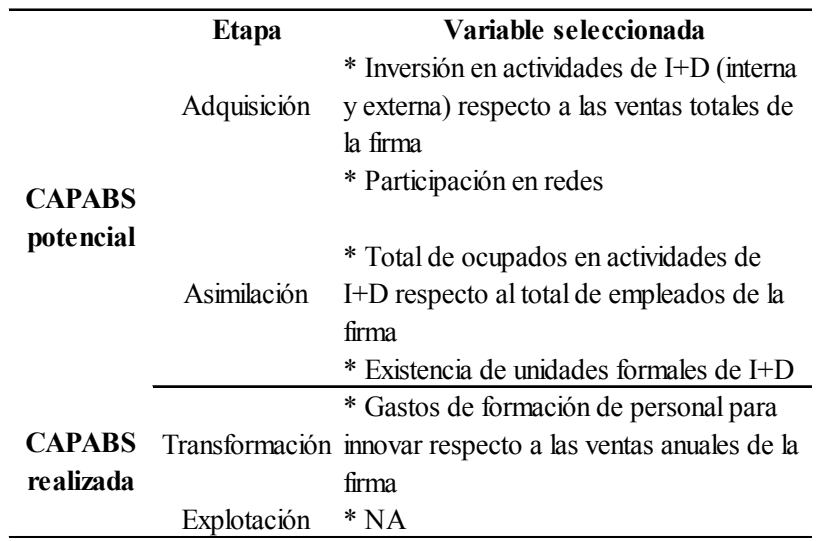

Fuente: Elaboración propia.

Por último, las variables de control seleccionadas fueron: presencia de capital extranjero, edad de la firma, ingresos por ventas por persona ocupada (como medida de tamaño de la firma) y si la firma es exportadora o no.

La formulación matemática del modelo logit se presenta en la siguiente ecuación:

$P($ INNOVA $=1)=E X P\left(\alpha_{t}+\sum \beta_{t j}\right.$
CARACT $_{t j}+\sum \Gamma_{t k}$ CAPABS $) /[1+E X P$
$\left(\alpha_{t}+\sum \beta_{t j}\right.$ CARACT $_{t j}+\sum \Gamma_{t k}$ CAPABS

Donde:

- INNOVA es el tipo de innovación realizada, donde i es: 1 . Innovación tecnológica en producto, 2. Innovación tecnológica en procesos, 3. Innovación en técnicas de organización, 4. Innovación en técnicas de comercialización.

- CARACT $_{\mathrm{j}}$ son las variables de control, donde j es: 1. Indica la presencia de capital extranjero en la firma, 2. Edad de la firma a 2015, 3. Ingresos por ventas sobre número de personas ocupadas en 2015, 4. Indica si la firma es exportadora.

- CAPABS $_{k}$ son las variables vinculadas a la Capacidad de Absorción de la firma, donde k es: 1 . Inversión en I+D (interma+externa)/ Ventas anuales, 2. Participación en redes, 3. Total de ocupados en actividades de $\mathrm{I}+\mathrm{D}$ respecto al total de empleados de la firma, 4. Existencia de unidades formales de I+D en la firma, 5. Gastos de formación de personal para innovar respecto al total de ventas de la firma.

La ecuación anterior representa la probabilidad de ocurrencia del evento (innovó o no innovó), dadas las características determinadas por las variables independientes para cada una de las firmas. 


\section{Aspectos de la capacidad de absorción que aumentan la probabilidad de que la empresa sea innovadora}

Se realizaron diferentes análisis descriptivos para poder caracterizar a las empresas de la encuesta y las variables utilizadas en el modelo econométrico. En la Tabla 2 se presentan variables que tratan de caracterizar a las empresas respecto a las innovaciones que realizan y otras características relevantes, tabla 2:

Tabla 2

Caracterización de las empresas de la encuesta

\begin{tabular}{lcccc}
\hline \multicolumn{1}{c}{ Característica seleccionada } & Producto & Proceso & Organización & Comercialización \\
\hline Empresas que innovan (\# y \%)* & $242(26,0 \%)$ & $311(33,4 \%)$ & $171(18,4 \%)$ & $88(9,5 \%)$ \\
Edad promedio de la empresa (años) & 34 & 33 & 33 & 34 \\
Tamaño promedio (cantidad de empleados) & 136 & 150 & 136 & 101 \\
Presencia de capital extranjero (\# y \%)\# & $42(17,4 \%)$ & $56(18,0 \%)$ & $31(18,1 \%)$ & $11(12,5 \%)$ \\
La firma es exportadora (\# y \%)\# & $120(49,6 \%)$ & $148(47,6 \%)$ & $73(42,7 \%)$ & $46(52,3 \%)$ \\
\hline
\end{tabular}

* sobre el total de empresas seleccionadas (931)

\# sobre el total de empresas que innovan en ese tipo de innovación

Fuente: Elaboración propia.

Los datos de la Tabla 2 permiten concluir que la innovación que más realizan las empresas seleccionadas son innovaciones en proceso, seguidos por innovaciones en producto, innovaciones en organización y por último innovaciones en comercialización. Por otra parte, estas empresas están consolidadas (con más de 30 años en el mercado), no presentan diferencias en la edad promedio por tipo de innovación realizada y tienen un tamaño considerable (ya que en Uruguay las empresas de más de 100 empleados son consideradas grandes). También, se puede observar que el porcentaje de empresas que presentan capital extranjero entre las empresas que innovan es relativamente bajo y que en ninguno de los tipos de innovación supera el $20 \%$. Otro dato importante es que un porcentaje muy importante de empresas que han innovado en alguno de los tipos de innovación considerados, son firmas que son exportadoras (por encima de un mínimo de $40 \%$ ).

En lo que respecta a las variables que se seleccionaron para medir la capacidad de absorción se clasificaron en cualitativas (Participación en redes y Existencia de unidades formales de I+D) y variables cuantitativas (que son las restantes variables utilizadas). Las medidas de asociación, prueba Chi cuadrado ( $x 2)$ y Coeficiente de contingencia (C), se calcularon para el cruce de las variables de innovación con las variables cualitativas que componen la capacidad de absorción. 
Se encontró que hay asociación entre el tipo de innovación y la participación en redes en todos los casos y que la mayor asociación se observa concretamente entre Innovación en Proceso y Participación en redes, con valores de $X 2=27,592, P_{\text {_ }}$ valor $=0,000$ y $C=0,170$, $P_{\text {_valor }}=0,000$. $^{3}$ También se estudiaron las correlaciones entre las variables cuantitativas que se incluyen para medir la capacidad de absorción. Si bien se establece que la correlación es positiva, los valores observados son bajos lo que amerita que puedan ser incorporados en el modelo econométrico.

Respecto al análisis econométrico, se debe destacar que todos los modelos logit calculados tuvieron una muy buena bondad de ajuste, ya que cada uno de ellos estimaron bien, como mínimo el $75 \%$ de las observaciones, nivel que se considera más que aceptable. Lo anterior quiere decir, que el conjunto de variables seleccionadas contribuyó significativamente a explicar la probabilidad de que las empresas hayan realizado innovaciones. Seguidamente se detallan los resultados de cada uno de los cuatro modelos: 4

En la Tabla 3 se presentan los resultados obtenidos. ${ }^{4} \mathrm{Si}$ se pone foco en los resultados referidos a las Innovaciones tecnológicas en Producto se puede observar que mientras que la participación en redes no parece incidir, las demás variables que tratan de captar la capacidad de absorción impactan en forma positiva y significativa.

3 Las pruebas estadísticas están a disposición del lector, alcanza con contactar a los autores.

4 Es importante tener en cuenta que, en un modelo logit los coeficientes estimados no tienen la misma interpretación que los coeficientes estimados de los modelos lineales. En los modelos lineales, los coeficientes miden los efectos de los cambios unitarios en cualquier variable explicativa sobre la variable a ser explicada y es una relación constante. En cambio, el modelo logit es un modelo no lineal y la influencia sobre la probabilidad de que la empresa sea o no innovadora derivada de cambios unitarios en las variables explicativas ya no es constante, o sea el coeficiente de la variable que se considere no mide el efecto parcial, por lo cual no se focaliza el análisis en la interpretación de los valores de los coeficientes, sino en los cálculos de probabilidad.

$5 \quad$ Para poder interpretar los resultados presentados se tiene que comprender adecuadamente los estadísticos que permiten demostrar la bondad de ajuste de los modelos pues, aunque reciben el nombre de $\mathrm{R}$ cuadrado, no tienen el mismo significado que en la regresión lineal. Los datos presentados en la Tabla 3 muestran valores para estos estadísticos, que son superiores o similares a los encontrados en otras investigaciones que utilizan modelos econométricos semejantes. Por ejemplo, los modelos logit en Rodil, Vence y Sánchez (2016) y tobit en Filipescu, Prashantham, Rialp y Rialp (2013). La prueba de significación de Hosmer y Lemeshow muestra valores superiores al $5 \%$, por lo que se puede concluir que la regresión presenta niveles aceptables de ajuste con respecto a los datos observados en todos los modelos. 


\section{Tabla 3 \\ Capacidad de Absorción vs Innovación}

\begin{tabular}{|c|c|c|c|c|c|c|c|c|}
\hline & \multicolumn{2}{|c|}{$\begin{array}{c}\text { Innovación } \\
\text { tecnológica en } \\
\text { Producto }\end{array}$} & \multicolumn{2}{|c|}{$\begin{array}{c}\text { Innovación } \\
\text { tecnológica en } \\
\text { Proceso }\end{array}$} & \multicolumn{2}{|c|}{$\begin{array}{c}\text { Innovación en } \\
\text { técnicas de } \\
\text { Organización }\end{array}$} & \multicolumn{2}{|c|}{$\begin{array}{c}\text { Innovación en } \\
\text { técnicas de } \\
\text { Comercialización }\end{array}$} \\
\hline & $\operatorname{Exp}(B)$ & Sig. & $\operatorname{Exp}(B)$ & Sig. & $\operatorname{Exp}(B)$ & Sig. & $\operatorname{Exp}(B)$ & Sig. \\
\hline Presencia de capital extranjero & 1,006 & & 1,111 & & 1,281 & & 0,516 & \\
\hline Antigüedad & 1,009 & $* *$ & 1,008 & $* *$ & 1,006 & & 1,005 & \\
\hline Facturación por personal ocupado & 1,000 & & 1,000 & & 1,000 & & 1,000 & \\
\hline Exportaciones & 1,369 & & 1,509 & & 0,990 & & 1,707 & $* *$ \\
\hline Inversión en $\mathrm{I}+\mathrm{D}$ sobre ventas totales & 1,586 & $* * *$ & 0,986 & & 0,950 & & 0,896 & \\
\hline Participación en redes & 1,345 & & 2,064 & $* * *$ & 1,458 & & 2,260 & $* *$ \\
\hline Ocupados en I+D sobre ocupados totales & 1,080 & $* * *$ & 1,186 & $* * *$ & 1,038 & $* * *$ & 1,043 & $* * *$ \\
\hline Existencia de unidades formales de $\mathrm{I}+\mathrm{D}$ & 6,287 & $* * *$ & 4,179 & $* * *$ & 3,817 & $* * *$ & 4,022 & $* * *$ \\
\hline Gastos en formación en I+D sobre ventas totales & 40,731 & $* *$ & 0,527 & $* *$ & 1,313 & & 3,611 & $* *$ \\
\hline Constante & 0,131 & $* * *$ & 0,225 & $* * *$ & 0,137 & $* * *$ & 0,052 & $* * *$ \\
\hline \multicolumn{9}{|l|}{ Estadísticos } \\
\hline R cuadrado de Cox y Snell & \multicolumn{2}{|c|}{0,223} & \multicolumn{2}{|c|}{0,172} & \multicolumn{2}{|c|}{0,070} & \multicolumn{2}{|c|}{0,076} \\
\hline R cuadrado de Nagelkerke & \multicolumn{2}{|c|}{0,326} & \multicolumn{2}{|c|}{0,238} & \multicolumn{2}{|c|}{0,114} & \multicolumn{2}{|c|}{0,163} \\
\hline Sig. Prueba de Hosmer y Lemeshow & \multicolumn{2}{|c|}{0,107} & \multicolumn{2}{|c|}{0,234} & \multicolumn{2}{|c|}{0,056} & \multicolumn{2}{|c|}{0,054} \\
\hline Porcentaje global de casos correctamente estimado (cuenta R cuadrado) & \multicolumn{2}{|c|}{0,824} & \multicolumn{2}{|c|}{0,753} & \multicolumn{2}{|c|}{0,814} & \multicolumn{2}{|c|}{0,909} \\
\hline
\end{tabular}

Fuente: Elaboración propia

Se puede observar que aquellas empresas de la industria manufacturera que más invierten en I+D, que cuentan con mayor cantidad de personas ocupadas en estas actividades y que más gastan en su formación, y que además tienen en su estructura organizativa una unidad formal dedicada a $\mathrm{I}+\mathrm{D}$, tienen una mayor probabilidad de innovar en producto.

Respecto a los resultados referidos a las Innovaciones tecnológicas en Proceso se puede afirmar que tienen una mayor probabilidad de innovar en proceso, aquellas empresas de la industria manufacturera que participan en redes, con mayor cantidad de personas ocupadas en $I+D$ y que tienen en su estructura organizativa una unidad formal dedicada a I+D. Las variables de la capacidad de absorción que no parecen incidir en que una firma realice este tipo de innovación son las relacionadas a inversiones en I+D y/o a gastos de formación.

Los resultados respecto a la Innovación en técnicas de Organización indican que sólo dos de las variables que miden la capacidad de absorción impactan significativamente en la probabilidad de que una empresa de este sector de la economía sea innovadora. Éstas son las relacionadas a ocupados en I+D y a la existencia de unidades formales de I+D (ver Tabla 3).

Los resultados muestran también que tienen una mayor probabilidad de innovar en técnicas de organización aquellas empresas de la industria manufacturera que tienen en su estructura organizativa una unidad formal dedicada a I+D y que por lo tanto poseen una mayor cantidad de personas ocupadas en I+D.

Por último, en el caso de la Innovación en técnicas de 
Comercialización solo la inversión en I+D no impacta en la probabilidad de realizar dicha innovación. Las demás variables de capacidad de absorción si presentan un impacto significativo (ver Tabla 3). Resulta así que, las empresas de la industria manufacturera que tienen participación en redes cuentan con una mayor cantidad de personas ocupadas en I+D, cuentan en su estructura organizativa con una unidad formal dedicada a I+D y que más gastan en formación de personal en $I+D$, tienen una mayor probabilidad de innovar en técnicas de comercialización.

En la Tabla 4 se presenta un resumen de los resultados encontrados por tipo de innovación. En dicha tabla se puede observar que las dos variables que se repiten para todos los tipos de innovación son: Existencia de unidades formales de I+D y Ocupados en I+D sobre ocupados totales en la firma. Es decir que, para el caso de las empresas manufactureras industriales en Uruguay, la creación de unidades especializadas en $\mathrm{I}+\mathrm{D}$ y una mayor proporción de personal en dichas tareas, se constituyen en aspectos importantes a la hora de incrementar la probabilidad de que la empresa haya realizado algún tipo de innovación.

\section{Tabla 4}

Resumen de resultados por tipo de innovación

\begin{tabular}{|c|c|c|c|c|c|}
\hline \multirow[b]{2}{*}{ Etapa } & \multirow[b]{2}{*}{ Variable } & \multicolumn{4}{|c|}{ Tipo de innovación } \\
\hline & & Producto & Proceso & Organización & Comercialización \\
\hline \multirow[t]{2}{*}{ Adquisición } & $\begin{array}{l}\text { Inversión en I+D sobre ventas } \\
\text { totales }\end{array}$ & $\sqrt{ }$ & & & \\
\hline & Participación en redes & & $\sqrt{ }$ & & $\sqrt{ }$ \\
\hline \multirow{2}{*}{ Asimilación } & $\begin{array}{l}\text { Ocupados en I+D sobre } \\
\text { ocupados totales }\end{array}$ & $\sqrt{ }$ & $\sqrt{ }$ & $\sqrt{ }$ & $\sqrt{ }$ \\
\hline & $\begin{array}{l}\text { Existencia de unidades formales } \\
\text { de I+D }\end{array}$ & $\sqrt{ }$ & $\sqrt{ }$ & $\sqrt{ }$ & $\sqrt{ }$ \\
\hline Transformaciór & $\begin{array}{l}\text { Gastos en formación en I+D sobre } \\
\text { ventas totales }\end{array}$ & $\sqrt{ }$ & & & $\sqrt{ }$ \\
\hline
\end{tabular}

Nota: el signo significa que la variable considerada impacta de forma positiva y significativa en la probabilidad de que la empresa realice la innovación considerada.

Fuente: Elaboración propia

A partir de las estimaciones de los parámetros del modelo logit, es posible calcular diferentes probabilidades de que una empresa sea innovadora. Para poder calcular estas probabilidades las variables cuantitativas se sustituyen por su valor promedio respectivo en la muestra de análisis y las variables cualitativas por el valor 1 si presentan la característica correspondiente 0 si no la presentan. Para calcular las probabilidades de que una firma sea innovadora se tomaron en cuenta las variables que fueron significativas al $5 \%$ y que tuvieran un Odd Ratio (Exp (B)) mayor que 1.

En la Tabla 5 se puede observar que la incidencia de las diferentes 
variables que afectan a la probabilidad de que una empresa sea innovadora, para todos los tipos de innovación, es importante. Esto parece evidente, pero la razón detrás de eso es que el valor de los coeficientes de esas variables en la respectiva ecuación de regresión resultó de signo positivo.

\section{Tabla 5}

\section{Aumento de la probabilidad de que una firma sea innovadora si se presenta la variable CAPAB según tipo de innovación (en puntos porcentuales)}

\begin{tabular}{|c|c|c|c|c|c|}
\hline \multirow[b]{2}{*}{ Etapa } & \multirow[b]{2}{*}{ Variable } & \multicolumn{4}{|c|}{ Tipo de innovación } \\
\hline & & Producto & Proceso & Organización & Comercialización \\
\hline \multirow[t]{2}{*}{ Adquisición } & $\begin{array}{l}\text { Inversión en I+D sobre ventas } \\
\text { totales }\end{array}$ & 19,3 & & & \\
\hline & Participación en redes & & 46,6 & & 13,0 \\
\hline \multirow{2}{*}{ Asimilación } & $\begin{array}{l}\text { Ocupados en I+D sobre } \\
\text { ocupados totales }\end{array}$ & 17,9 & 34,6 & 14,9 & 7,9 \\
\hline & $\begin{array}{l}\text { Existencia de unidades formales } \\
\text { de I+D }\end{array}$ & 57,9 & 63,9 & 40,1 & 22,7 \\
\hline Transformaciór & $\begin{array}{l}\text { Gastos en formación en I+D } \\
\text { sobre ventas totales }\end{array}$ & 17,8 & & & 7,6 \\
\hline
\end{tabular}

Fuente: Elaboración propia

Para todos los tipos de innovación, la variable de capacidad de absorción que impacta en forma más relevante en que una empresa sea innovadora es la existencia de unidades formales de I+D. Para la innovación tecnológica en productos el aumento de la probabilidad de que la empresa sea innovadora es de 57,9 puntos porcentuales (p.p.), para la innovación tecnológica es de 63,9 p.p., para la innovación en técnicas de organización es de 40,1 p.p. y en la innovación en técnicas de comercialización es de 22,7 p.p.

Para los casos de innovación tecnológica en proceso e innovación en técnicas de comercialización, la participación en redes aumenta la probabilidad de que una empresa sea innovadora, pero en menor medida que para la variable anterior, teniendo una mayor incidencia en el primer tipo de innovación que en el segundo $(46,6 \%$ p.p. y 13,0 p.p. respectivamente).

Por su parte, la inversión en I+D, medida respecto a las ventas totales de la firma, tiene incidencia solo en el caso de la innovación tecnológica en producto, aumentando la probabilidad de que sea innovadora en 19,3 p.p. Los ocupados en I+D, medida respecto al total de ocupados de la firma, aumentan la probabilidad de que sea innovadora en 17,9 p.p., para el caso de la innovación tecnológica en producto, en 34,6 p.p. para el caso de la innovación tecnológica en proceso el caso de la innovación en técnicas de organización y de 7,9 p.p. para el caso de la innovación en técnicas de comercialización. Esta variable tiene 
una incidencia mayor para el caso de la innovación en proceso.

Por último, los gastos en formación en I+D, medidos sobre las ventas totales de la firma aumentan la probabilidad de que una firma sea innovadora en producto en 17,8 p.p. y de 7,6 p.p. para el caso de la innovación en técnicas de comercialización.

\section{Conclusiones}

El objetivo de esta investigación se ha orientado a determinar si la capacidad de absorción, que es posible verificar a nivel de las empresas industriales en Uruguay, aumenta la probabilidad de que la empresa sea innovadora. Los resultados obtenidos ponen de manifiesto la importancia que tienen determinadas variables que miden la capacidad de absorción, para explicar la probabilidad de que una firma haya realizado innovaciones. Dicha conclusión general está en línea con lo detallado en la literatura revisada.

En cuanto a conclusiones más específicas, se debe de hacer notar que las dos variables que aparecen incidiendo en la probabilidad de que una empresa de la industria manufacturera uruguaya sea innovadora, y que se repiten para todos los tipos de innovación, son la existencia de unidades formales de I+D en la firma y el peso relativo que tiene el conjunto de empleados dedicados a I+D en relación con el total de empleados de la empresa. Estas dos variables están asociadas a la etapa de asimilación, una de las etapas que la literatura describe del concepto "capacidad de absorción", indicando que se trata, el proceso de asimilación, de una etapa significativa en el proceso de innovación, en la cual se generan rutinas y procesos que permiten analizar, procesar, interpretar y comprender la información externa obtenida, y transformarla luego en diferentes tipos de innovación.

Si se observa, por otra parte, la influencia de las variables que se utilizaron para describir la capacidad de absorción, sobre los tipos de innovación realizados, se comprobó que para el caso de la innovación tecnológica en producto son importantes variables que abarcan todas las etapas del concepto de capacidad de absorción utilizado, es decir las etapas de adquisición, de asimilación y de transformación. Lo mismo sucede para el caso de las innovaciones en técnicas de comercialización. Ello estaría indicando que las innovaciones en producto o comercialización que realizan las empresas industriales en Uruguay son las que más se benefician de la mayor capacidad de absorción, pudiendo identificar más y mejores conocimientos o explotarlos de una forma más eficiente.

En cambio, para el caso de las innovaciones tecnológicas en proceso y en técnicas de organización, los resultados mostraron que las variables que afectan la probabilidad de que una empresa realice esos tipos de innovación están relacionadas solamente a las etapas de adquisición y de asimilación, es decir, las etapas más tempranas en el proceso de innovación. Esta conclusión se alinea con varias investigaciones analizadas, que muestran a estas etapas tempranas como significativas en lo que respecta a la capacidad de absorción potencial de la empresa.

Otro aspecto para destacar se refiere al hecho de que la inversión en I+D solo aumenta la probabilidad de que una empresa de la industria manufacturera uruguaya sea innovadora, para el caso de la innovación tecnológica en producto, resultado que parece lógico para este 
tipo de innovación que en principio tiene asociado un componente de ciencia y tecnología más acentuado que el resto. En cambio, la participación en redes, la otra variable relacionada con la etapa de adquisición de conocimientos influye solo en los casos de la innovación tecnológica en procesos y en la innovación en técnicas de comercialización.

Un aspecto que resulta interesante y que debe resaltarse, es que de todas las variables relacionadas a la capacidad de absorción que se consideraron para calcular las probabilidades, y para todos los tipos de innovación, es la Existencia de unidades formales de $I+D$, la variable que más contribuye a subir la probabilidad de que la empresa sea innovadora, variable que está relacionada a la etapa de asimilación. Se destaca la mayor incidencia para el caso de la innovación tecnológica en proceso, seguida por la innovación tecnológica en producto. Este resultado es diferente al que encontraron otros autores que concluyen que la etapa que más influye en su estudio es la de adquisición ${ }^{6}$. Tal vez eso sea así por tratarse en el caso de esa investigación, de empresas de países desarrollados, donde las variables de esta etapa están en un estadio más avanzado de desarrollo, con una mayor inversión en $\mathrm{I}+\mathrm{D}$ relativa y de participación en redes para innovar. Los resultados encontrados estarían demostrando la importancia de institucionalizar las actividades de innovación en la organización de la empresa y refuerza la idea de que la etapa de asimilación de la capacidad de absorción es fundamental para el caso de la innovación en las empresas manufactureras industriales en Uruguay. Esto debería de servir como una guía para las diferentes organizaciones, tanto del gobierno como empresariales, a la hora de implementar políticas de incentivos a la innovación empresarial.

Por último, es necesario explicitar que una limitación de la presente investigación se relaciona con el hecho de que no se consideraron en los diferentes modelos, por falta de datos, variables que pudieran captar la etapa de explotación del concepto capacidad de absorción. Futuras investigaciones deberían de tenerlas en cuenta.

\section{Referencias Bibliográficas}

Aguilera, R. V., Ciravegna, L., CuervoCazurra, A. y González-Pérez, M. A. (2017). Multilatinas and the internationalization of Latin American firms. Journal of World Business, 52, 447-460. https://doi.org/10.1016/j. jwb.2017.05.006

Ali, M. y Park, K. (2017). The mediating role of an innovative culture in the relationship between absorptive capacity and technical and nontechnical innovation. Journal of Business Research, 69(5) 1669$1675 . \quad$ https://doi.org/10.1016/j. jbusres.2015.10.036

Ali, M., Seny Kan, K. A. y Sarstedt, M. (2016). Direct and configurational paths of absorptive capacity and organizational innovation to successful organizational performance. Journal of Business Research, 69, 5317-5323. https://doi. org/10.1016/j.jbusres.2016.04.131

ANII (2015). Encuesta de actividades de innovación en la industria

\footnotetext{
6 Por ejemplo, Expósito-Langa et al, (2011).
} 
manufacturera

seleccionados

$$
\mathrm{y}
$$

$(2010$

servicios -2012).

Principales resultados. Colección Indicadores y Estudios Nr. 9. Agencia Nacional de Investigación e Innovación. Montevideo. Agosto 2015.

BCU (2019). Cuentas Nacionales. Banco Central del Uruguay. Montevideo. https://bit.ly/3ndObGK

Belderbos, R., Carree, M., Diederen, B., Lokshin, B. y Veugelers, R. (2004). Heterogeneity in R\&D cooperation strategies. International journal of industrial organization, 22(8), 12371263.

Brenes, E. R., Camacho, A. R., Ciravegna, L. y Pichardo, C. A. (2016). Strategy and innovation in emerging economies after the end of the commodity boom-Insights from Latin America. Journal of Business Research, 69(10), 4363$4367 . \quad$ https://doi.org/10.1016/j. jbusres.2016.03.059

Calero-Medina, C. y Noyons, E. C. (2008). Combining mapping and citation network analysis for a better understanding of the scientific development: The case of the absorptive capacity field. Journal of Informetrics, 2(4), 272-279. https:// doi.org/10.1016/j.joi.2008.09.005

Camacho, M., Jung, A., Horta, R. y García, S. (2010). ¿Cómo innovan las empresas exitosas en Uruguay? Una aplicación del modelo "la cometa de la innovación". Instituto de Competitividad. Universidad Católica del Uruguay. Montevideo.

Carpio, C. R. y Afcha, S. M. (2020). Efecto de las barreras de innovación en la capacidad de absorción de las empresas innovadoras. Revista de Métodos Cuantitativos para la Economía y la Empresa, 30, 3-22. https://doi.org/10.46661/ revmetodoscuanteconempresa.2940

Cheng, L. y Tao, Z. (1999). The impact of public policies on innovation and imitation: The role of R\&D technology in growth models. International Economic Review, 40(1), 187-207.

Chen, S. T. y Chang, B. G. (2012). The effects of absorptive capacity and speed on organizational innovation: a study of organizational structure as an antecedent variable. Contemporary Management Research, 27. https:// doi.org/10.7903/cmr.7996

Cepeda-Carrión, G., Cegarra-Navarro, J. y Jiménez-Jiménez, D. (2012). The effect of absorptive capacity on innovativeness: Context and information systems capability as catalysts. British Journal of Management, 23, 110-129.

Cohen, W. M. y Levinthal, D. A. (1989). Innovation and Learning: the two faces of R\&D. Economic Journal, 99, 569-596.

Cohen, W. M. y Levinthal, D. A. (1990). Absorptive Capacity: A new Perspective on Learning and Innovation. Administrative Science Quarterly, 35, 128-152.

De Macedo, Soares, T. D. L., Silva Barboza, T. y de Oliveira, Paula F. (2016). Absorptive Capacity, Alliance Portfolios and Innovation Performance: an Analytical Model Based on Bibliographic Research. Journal of Technology Management y Innovation, 11(3), 2132. https://doi.org/10.4067/S071827242016000300003

Del Carpio Gallegos, J. F. y Miralles Torner, F. (2018). Absorptive capacity and innovation in low-tech companies in emerging economies. Journal of Technology Management y Innovation, 13(2), 3-10. 
Dyer, J. y Singh, H. (1998). The relational view: Cooperative strategies and sources of interorganizational competitive advantage. Academy of Management Review, 23(4), 660679.

Escribano, A., Fosfuri, A. y Tribó, J. (2009). Managing external knowledge flows: The moderating role of absorptive capacity. Research Policy, 38, 96-105.

Expósito-Langa, M., Molina-Morales, F. y Capo-Vicedo, J. (2011). New Product Development and Absorptive Capacity in Industrial Districts: A Multidimensional Approach. Regional Studies, 45(3), 319-331.

Fabrizio, K. R. (2009). Absorptive capacity and the search for innovation. Research Policy 38, 255267.

Filipescu, D. A., Prashantham, S., Rialp, A. y Rialp, J. (2013). Technological Innovation and Exports: Unpacking Their Reciprocal Causality. Journal of International Marketing, 21(1), 23-38.

Giuliani, E. (2005). Cluster absorptive capacity: Why do Some Clusters Forge Ahead and Others Lag Behind? European Urban and Regional Studies, 12, 269-288.

González-Campo, C. H. y Hurtado Ayala, A. (2014). Influencia de la capacidad de absorción sobre la innovación: un análisis empírico en las mipymes colombianas. Estudios Gerenciales, 30, 277-286.

Heijs, J. (2010). Política tecnológica, aprendizaje y capacidad de absorción de conocimientos: los círculos viciosos y virtuosos. Innovación a aprendizaje: lecciones para el diseño de políticas. Parrilli, Coordinador, Innobasque, 324-350.

Horta, R., Silveira, L. y Camacho, M.
(2015). Competitividad e innovación en la industria manufacturera en el Uruguay. Revista CTS, 28(10), 2349.

Horta, R., Silveira, L. y Francia, H. (2020). Innovaciones y exportaciones: una nueva perspectiva de estudio de la industria manufacturera uruguaya. Estudios Gerenciales, 36(157), 402$414 . \quad$ https://doi.org/10.18046/j. estger.2020.157.3685

Jansen, J., Van den Bosch, A. J. y Volberda, H. W. (2005). Managing Potential and Realized Absorptive Capacity: How Do Organizational Antecedents Matter? Academy of Management Journal, 48(6), 9991015.

Jong, J. y Freel, M. (2010). Absorptive capacity and the reach of collaboration in high technology smalls firms. Research Policy, 39, 47-54.

Kostopoulos, K., Papalexandris, A., Papachroni, M. y loannou, G. (2011). Absorptive capacity, innovation, and financial performance. Journal of Business Research, 64(12), 13351343. $\quad$ https://doi.org/10.1016/j. jbusres.2010.12.005

Lane, P. J., Koka, B. R. y Patthak, S. (2006). The reification of absorptive capacity: a critical review and rejuvenation of the construct. Academy of Management Review, 31(4), 833-863.

Lane, P. J. y Lubatkin, M. (1998). Relative absorptive capacity and interorganizational learning. Strategic Management Journal, 19, 461-477.

Lazaric, N., Longhi, C. y Thomas, C. (2008). Gatekeepers of knowledge versus Platforms of Knowledge: From Potential to Realized Adsorptive Capacity. Regional Studies, 42(6), 


\section{7-852}

Leal-Rodríguez, A. L., Roldán, J. L., Ariza-Montes J. A. y Leal-Millán, A. (2014). From potential absorptive capacity to innovation outcomes in project teams: The conditional mediating role of the realized absorptive capacity in a relational learning context. International Journal of Project Management, 32(6), 894-907. https://doi.org/10.1016/i. ijproman.2014.01.005

Lugones, G., Suarez, D. y Gregorini, S. (2007). La Innovación como fórmula para mejoras competitivas compatibles con incrementos salariales. Evidencias en el caso argentino. Documento de Trabajo 36. Centro de Estudios sobre Ciencia, Desarrollo y Educación Superior. Argentina.

Luo, Y. (1997). Partner selection and venturing success: The case of joint ventures with firms in the People's Republic of China. Organization Science, 8(6), 648-662.

Maynez-Guaderrama, A., CavazosArroyo, J. y Nuño-de la Parra, J. (2012). La influencia de la cultura organizacional y la capacidad de absorción sobre la transferencia de conocimiento tácito intra-organizacional. Estudios Gerenciales, 28, 191-211.

Morrison, A. (2008): Gatekeepers of Knowledge within Industrial Districts: Who They Are, How They Interact. Regional Studies, 42(6), 817-835.

Mowery, D. y Oxley, J. (1995). Inward technologies transfer and competitiveness: The role of national innovation systems. Cambridge Journal of Economics, 19(1), 67-93.

Nelder, J. A. y Wedderburn, W. M. (1972). Generalized Linear Models. Journal of the Royal Statistical Society,
135(3), 370-384.

OCDE (2005). Manual de Oslo. Guía para la recogida e interpretación de datos sobre innovación, $3^{a}$ edición. Organización para la Cooperación y el Desarrollo Económicos. https:// dx.doi.org/10.1787/9789264065659$\underline{\text { es }}$

Oltra, M. J. y Flor, M. (2003). The impact of technological opportunities and innovative capabilities on firms' output innovation. Creativity and Innovation Management, 12(3), 137144.

Petroni, A. y Panciroli, B. (2002). Innovation as a determinant of suppliers' roles and performances: an empirical study in the food machinery industry. European Journal of Purchasing y Supply Management, 8(3), 135-149.

Ramadhan, M., Hussain, A. y al-Hajji, R. (2013). Limitations of Kuwait's economy: Anabsorptive capacity perspective. Modern Economy, 4, 412-417.

Rangus, K. y Slavec, A. (2017). The interplay of decentralization, employee involvement and absorptive capacity on firms' innovation and business performance. Technological Forecasting and Social Change, 120, 195-203. https://doi.org/10.1016/j. techfore.2016.12.017

Rodil, Ó., Vence, X. y Sánchez M. C. (2016). The relationship between innovation and export behaviour: The case of Galician firms. Technological Forecasting y Social Change, 113, 248-265. https://doi.org/10.1016/j. techfore.2015.09.002.

Rodríguez, G., García, R., Cervera, J., Sanabria. N., y Niebles, E. E. (2017). Capacidad de absorción e innovación: Un análisis para la Industria en Colombia. 1 edición. Barranquilla, 
Sello Editorial Uniautónoma.

Rodríguez, G., Sanabria, N., Reyes, A., Ochoa, A. y Altamar, L. (2017). Análisis de la capacidad de absorción en la empresa: Una revisión de literatura. Semestre Económico, 20(43), 139-160.

Sánchez, M. I. y Acosta, B. F. (2020). Capacidad de absorción: Integración estratégica entre aprendizaje tecnológico, resiliencia y competitividad empresarial. Revista Venezolana de Gerencia, 25(4), 528-547. https://www. produccioncientificaluz.org/index. php/rvg/article/view/35208

Schmidt, T. y Rammer, C. (2006). The determinants and effects of technological and nontechnological innovations-Evidence from the German CIS IV. Zentrum fur Europaische Wirtschaftsforschung (ZEW), Mannheim.

Solís, S. Y., Zerón, Félix, M. y Sánchez Tovar, Y. (2019). Efectos de la Capacidad de Absorción en la Innovación del sector Industrial en el Norte de México. Nova Scientia, 23(11), 447-472. https://doi. org/10.21640/ns.v11i23.2039

Stock, G., Greis, N. y Fischer, W. (2001). Absorptive Capacity and New Product Development. The Journal of High Technology Management Research, 12(1), 77-91.

Tsai, W. (2001). Knowledge transfer in interorganizational networks: effects of network position and absorptive capacity on business unit innovation and performance. Academy of Management Journal, 44(5), 9961004

Vega-Jurado, J., Fernández, I., Gutiérrez, A. y Manjarres, L. (2005). Los determinantes de la innovación tecnológica en la empresa: una aproximación a través del concepto de capacidad de absorción. ALTEC; 2005. XI Seminario Latino Iberoamericano de Gestión Tecnológica. 25-28 de octubre de 2005.

Vermeulen, H. (2004). Models and modes of immigrant integration and where does southern Europe fit? In C. Inglessi, A. Lyberaki, H. Vermeulen, y G. J. van Wijngaarden (Eds.), Immigration and Integration in Northern versus Southern Europe. Athens: Netherlands Institute in Athens.

Visser, E. J. y Atzema, O. (2008). With or Without Clusters: Facilitating Innovation through a Differentiated and Combined Network Approach. European Planning Studies, 16(9), 1169-1188.

Volverda, H. W., Foss, N. y Lyles, A. (2010). Absorbing the Concept of Absorptive Capacity: How to Realize Its Potential in the Organization. Field Organization Science, 21(4), 931951.

Yaseen, S. G. (2020). Potential Absorptive Capacity, Realized Absorptive Capacity and Innovation Performance. En: Ahram T., Taiar R., Colson S., Choplin A. (Eds.) Human Interaction and Emerging Technologies. IHIET 2019. Advances in Intelligent Systems and Computing, 1018. Francia: Springer.

Zahra, S. A. y George, G. (2002). Absorptive Capacity: A review, reconceptualization, and extension. Academy of Management Review, 27(2), 185-203.

Zahra, S. A. y Hayton, J. C. (2008). The effect of international venturing on firm performance: the moderating influence of absorptive capacity. Journal of Business Venturing, 23(2), 195-220. 